\title{
An innovative mobile data collection technology for public health in a field setting
}

\author{
Shamir Mukhi ${ }^{*}$, Karna Dhiravani', Brad Micholson', Lin Yan', Jenna Hatchard1, Samira \\ Mubareka², Christopher Bergeron $^{3}$, Tim Beattie ${ }^{4}$
}

1. Canadian Network for Public Health Intelligence, National Microbiology Laboratory, Winnipeg, MB

2. Sunnybrook Research Institute, University of Toronto, Toronto, ON

3. First Nations and Inuit Health Branch Manitoba Region, Winnipeg, MB

4. Water and Air Quality Bureau, Health Canada, Ottawa, ON

\begin{abstract}
Objectives: The Canadian Network for Public Health Intelligence (CNPHI) is a secure, web-based scientific informatics and biosurveillance platform that leverages disparate public health information resources and expertise for the direct benefit of local, regional and national decision makers. CNPHI fosters collaboration and consultation through innovation in disease surveillance, intelligence exchange, research and response to protect, promote and support public health. The objective of this article is to present the CNPHI 'on the go' mobile application, and to discuss preliminary evaluation of the technology. The mobile application is intended to enable rapid mobile data collection using both online and offline modes supporting various stages of surveillance and response through the extension of data collection and analysis to the mobile environment.

Methods: Two needs assessment meetings were held with stakeholders representing individuals from government, academia and research institutions, to inform the development of the CNPHI "on the go" mobile application. An initial version of the mobile technology (an "app") was developed and piloted by end-users with expertise in the field. Two focused pilots were conducted to test the technology: Pilot 1: 17-7-2017 to 21-11-2017 (6 participants); Pilot 2: 25-7-2017 to 15-9-2017 (2 participants). An initial consultation was held with the project leads to identify data elements for mobile data collection. A custom data collection form was designed using CNPHI's Web Data technology for each pilot, which was then made available through the mobile app. The technology was assessed using feedback received during each pilot as well as through a survey that was conducted at the conclusion of pilots.

Results: Pilot participants reported that the mobile technology allowed seamless data collection, data management and rapid information sharing. Participants also reported that the entire process was seamless, simple, efficient, and that fewer steps were required for data collection and management. Further, significant efficiencies were gained by directly entering information using the mobile app without having to transfer handwritten information into an electronic database. An overall positive experience was reported by participants from both pilots.
\end{abstract}


Discussion: Literature suggests that traditional methods of surveillance and data collection using a paper based methodology pose many challenges such as data loss and duplication, difficulty in managing the database, and lack of timely access to the data. Accurate and rapid access is critical for public health professionals in order to effectively make decisions and respond to public health emergencies. Results show that the CNPHI "on the go" app is well poised to address some of the suggested challenges. A limitation of this study was that sample size for pilot participation was small for capturing overall feedback on the readiness of the technology for integration into regular surveillance activities and response procedures.

Conclusions: CNPHI "on the go" is a customizable technology developed within an already thriving collaborative CNPHI platform used by public health professionals, and performs well as a tool for rapid data collection and secure information sharing.

Keywords: Data collection, mobile, epidemiology, informatics, biosurveillance

Correspondence: shamir.mukhi@canada.ca

DOI: 10.5210/ojphi.v10i2.9114

Copyright @2018 the author(s)

This is an Open Access article. Authors own copyright of their articles appearing in the Online Journal of Public Health Informatics. Readers may copy articles without permission of the copyright owner(s), as long as the author and OJPHI are acknowledged in the copy and the copy is used for educational, not-for-profit purposes.

\section{Introduction}

Communication, collaboration and information sharing are critical for effective and efficient public health surveillance systems. Enhancements of the traditional approaches to data collection and reporting to support surveillance and response activities would allow public health professionals from different jurisdictions to effectively collaborate, communicate, and collect critical situational intelligence through a common platform at short notice. This requires secure and familiar tools, allowing flexible and rapid interventions through the generation and dissemination of public health intelligence.

Mobile phone and pad related technology has been or is being fully adopted in most communities in Canada and globally, for personal and professional communications. In Canada, $87 \%$ of the population owned a mobile phone in 2016 [1]. In addition to that, $77 \%$ of the population had a smart phone in 2016, which is 26\% higher than in 2012, demonstrating a rapidly increasing trend [1]. Advances in mobile technology have provided an opportunity to use it to inform surveillance activities related to the transmission of pathogenic microorganisms or other threats to public health and safety. The use of mobile technology for the purpose of data collection, information transmission and reporting provides a potential for real-time monitoring and early warning intelligence, alleviating the resource intensity traditionally required for indepth field investigations and paper based data collection methods [2].

Lack of rapid access to data poses challenges for effective and timely response during public health emergencies. Despite its significance, seamless data collection and management during 
public health emergencies has been problematic in the past [3]. The current method of using paper to collect the data is cumbersome and may lead to delays in making crucial decisions related to public health. The traditional surveillance and data collection method is also prone to other issues such as data duplication, data loss and errors during data entry into an electronic database. Timely and accurate data is important for public health personnel to effectively make decisions and to provide services. World Health Organization (WHO) has reported that despite all the benefits and high penetration rate, mobile technology is still not commonly used for the purpose of data collection, data transmission and reporting in public health [2].

The Canadian Network for Public Health Intelligence (CNPHI) was developed by Public Health Agency of Canada in 2004 to improve Pan-Canadian public health surveillance, communication and response through a comprehensive framework of applications and resources [4]. CNPHI is currently used by a large number of Federal, Provincial and Territorial public health professionals, including epidemiologists, lab scientists, physicians, nurses, animal health professionals, drinking water regulators, policy groups and provides an infrastructure for public health surveillance in Canada. CNPHI has continued to make significant progress in filling gaps in data collection, collation, analysis and reporting by leveraging and building upon its current infrastructure.

In 2014, CNPHI initiated a Canadian Safety and Security Program (CSSP) funded project 'CNPHI on the go' - intended to provide infrastructure to support the usage of mobile technology to foster bi-directional communications, support investigations, and generate geoaware biosurveillance intelligence related to human, animal, and environmental public health issues of concern. The project, since it is built on the existing CNPHI platform, benefits every stage of surveillance and response through the extension of data collection and analytical capacities through inter-jurisdictional and interdisciplinary collaboration.

The CNPHI on the go project focused on extending the CNPHI platform for rapid data collection and support for response related activities. This article focuses on the rapid data collection component, which is based on the extension of CNPHI's Web Data technology [5] on the mobile app and how it was used for the purpose of the mobile data collection in the field environment. Web Data is an innovative technology developed by CNPHI, which allows users to rapidly deploy secure and adaptable forms for collecting data. Web Data is an important technology in the context of response because it provides a platform for response teams to collect and analyze data in a systematic way. The web data technology has been tested during the 2009-2010 H1N1 influenza pandemic and has been in use since then by public health personnel in Canada [6].

Web Data technology extended on the mobile app allows users to create user-defined databases and surveys that can be used to collect data through open- and closed-ended questions, whether quantitative or qualitative in nature. Some of the key features of this technology include: a dynamic form builder, interactive queries/reports, a customizable data uploader and advanced analysis. It provides users with seamless access to data via a secure, web-based model with multiple levels of access, as well as jurisdictional and field level controls. The technology provides users with an ability to perform integrated search and interrogation and also an ability to produce instant reports [5]. We partnered with Sunnybrook Research Institute (SRI) and Health Canada, First Nations Inuit Health Branch - Manitoba Region (FNIHB-MB) to pilot the 
Web Data technology on the CNPHI on the go app. The pilots were carried out in field environments, which included remote areas where internet connectivity can be problematic.

\section{Method, Design and Development}

Prior to development of the mobile app, needs assessment meetings were held with a group of stakeholders to inform the development of CNPHI on the go. The meetings were attended by a multi-disciplinary team representing individuals from government, academia and research institutions. These stakeholders comprised an array of disciplines, including; epidemiologists, drinking water regulators, animal health veterinarians, public health inspectors, laboratory specialists and medical personnel. The meetings focused on identifying opportunities and challenges to support project implementation, and identifying gaps and additional capabilities that are required to make it operational. Some of the specific topics discussed during the meetings included brainstorming ideas for mobile data collection and offline data collection. An initial meeting was held in 2015 followed by a second meeting in 2016.

In summary, the discussions from these meetings identified the following key areas:

- Simplicity: Participants mentioned that the app should be simple to use. It is important that the users feel comfortable with the system and are able to easily enter the data and navigate the application;

- Bi-directional: The participants also mentioned that there needs to be a function that allows for a two-way communication between the field workers and administrators in close to real-time. Team members will therefore have an ability to exchange important information and disclose their location in a timely manner if required and appropriate;

- Offline: The participants also mentioned that the issues related to mobile phone coverage must be taken into account;

- Security: Issues related to security and user authentication need to be considered.

The CNPHI on the go comprises two main components:

- Mobile Infrastructure: This includes a dedicated mobile server that enables secure connectivity for mobile devices and integration with the existing CNPHI web platform through an interface with a user access management system and a business logic integration layer.

- Mobile Devices: This includes the mobile “app” specifically designed for mobile devices providing required functionality.

The CNPHI on the go app includes a multitude of features that extend specific CNPHI functionalities and facilitate seamless access for the users. Specifically:

- Mobile Web Data: The Web Data technology, which allows users to create the dynamic forms and surveys on CNPHI has now been extended to the mobile platform, allowing users to perform data collection from their mobile device. Once the data is submitted, the responses can be viewed under the Web Data interface on the web version. 
- Offline data submission: The forms are accessible once downloaded and would allow users to perform data collection and submission in the absence of connectivity. Once the data is saved within the app, records are queued for submission and automatically submitted to the web platform once stable connectivity is established.

- Access: The mobile platform uses the common CNPHI user access management system and can only be used by individuals who are registered users of the CNPHI platform.

- PIN code: The app allows users to set a 4 digit pin. The pin enables access to the app functionality regardless of the internet connectivity and also allows users to conveniently and quickly log-in instead of using their username and password. The pin is saved as long as the user does not sign out from the app.

- Geo-location: The app includes built-in automated capability to record latitude and longitude, if enabled by the user, before sending data to the web platform.

- Shared Device: The app is designed to allow possible sharing of a device by multiple personnel. This is achieved by the "remove stored data" functionality which erases all stored data including a pin, if enabled.

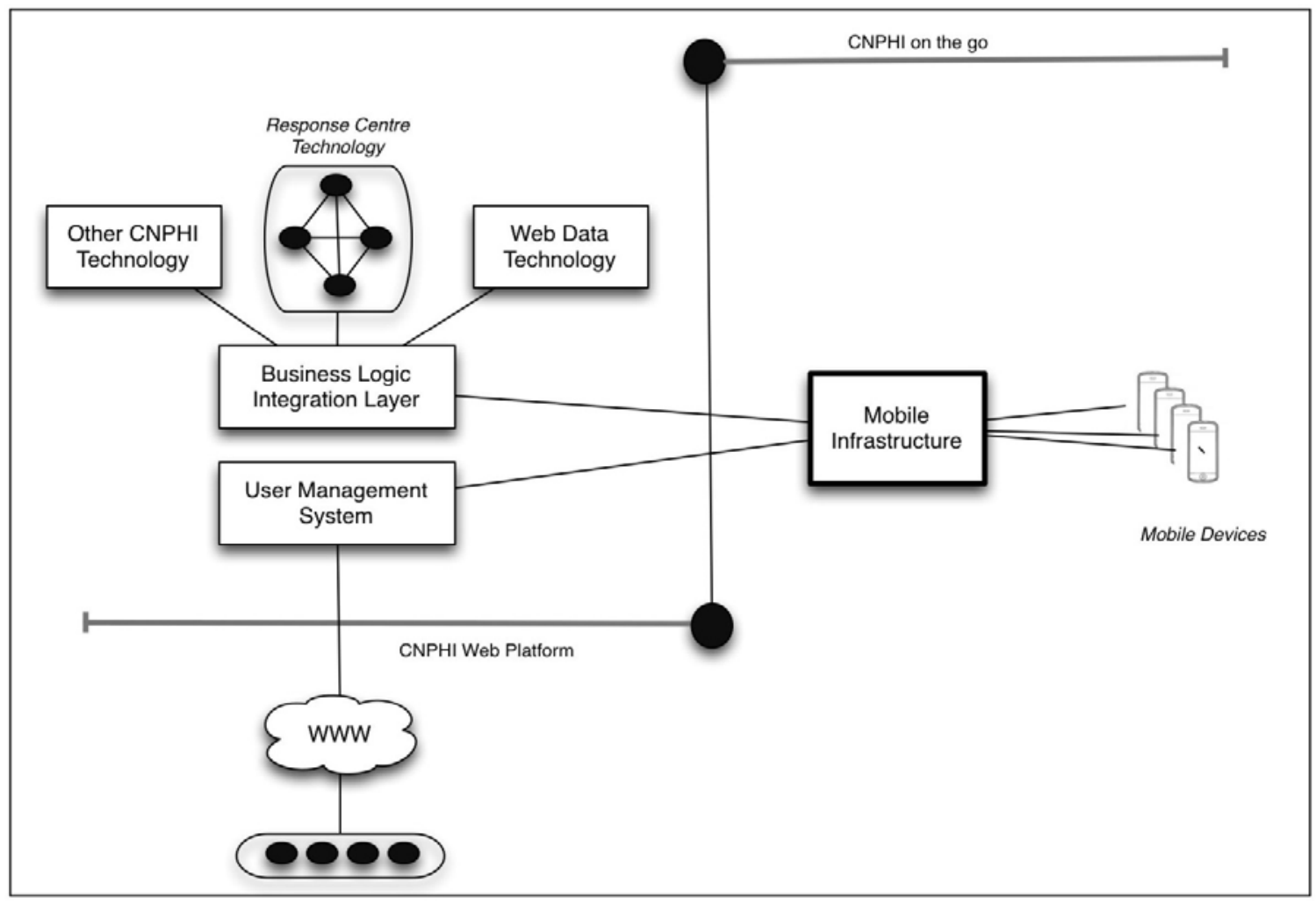

Figure 1: A high level view of the CNPHI on the go as an extension of the existing CNPHI platform. 


\section{Pilots: Deployment and Testing}

The initial version of the app ("alpha version") was developed on the Android platform and was piloted in partnership with two organizations in the summer of 2017 (FNIHB-MB: 17-7-2017 to 21-11-2017; SRI: 25-7-2017 to 15-9-2017).

FNIHB - MB led the first pilot. The group is responsible for monitoring drinking water systems in First Nations communities in Manitoba. While collecting samples for bacteriological testing, they also collect metadata including sampling times and locations, residual chlorine values, turbidity readings, and other parameters from drinking water systems. A custom form was designed for this pilot and made available via the app, which was used in the field by Environmental Health Officers (EHOs). The mobile app was piloted in remote areas and inside structures with limited internet connectivity in order to test data collection capabilities under true field conditions.

The second pilot was with SRI, who led a project to demonstrate the feasibility of environmental sampling to detect the influenza virus in swine barns. It is believed that environmental sampling techniques that prove to be practical and effective at detecting the influenza virus would momentously benefit public health efforts in disease surveillance. Similarly, this capability would also support the early identification of emerging zoonotic infectious diseases of public health significance. As part of the project, data was collected using environmental samplers in a swine barn. A custom data collection form was designed for this application using the Web Data technology, which was then made available through the mobile app. Sample data was entered on the android device and laboratory testing results were superimposed once they were available.

The objective of the pilots was first to familiarize users and to introduce them to CNPHI mobile technology and enable collection of sampling data from the field using the CNPHI on the go app.

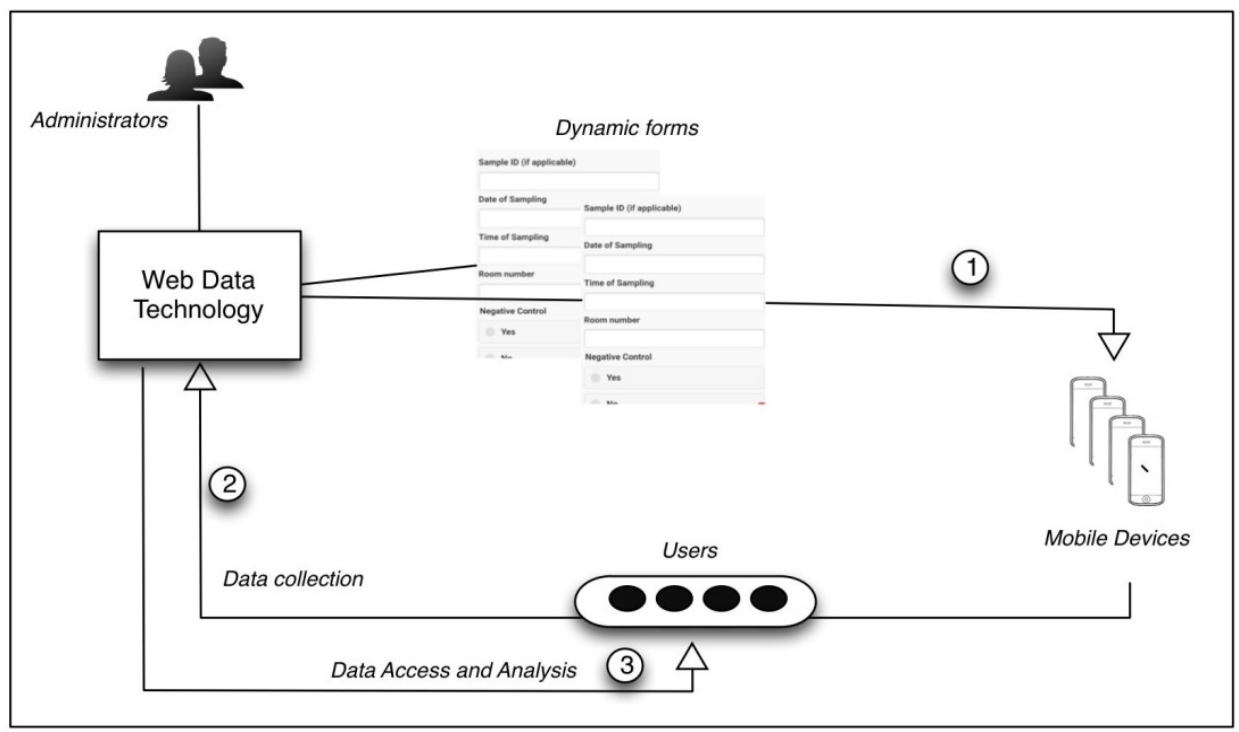

Figure 2: Data flow during the pilot phase. 
During the planning stages of the pilot, an initial consultation was held with the project leads to identify data elements for mobile data collection. Once the data elements were finalized, two forms were developed using the Web Data technology on the CNPHI web platform. The forms consisted of numerous fields including checkboxes, radio buttons and drop-down lists, among other selection and entry strategies.

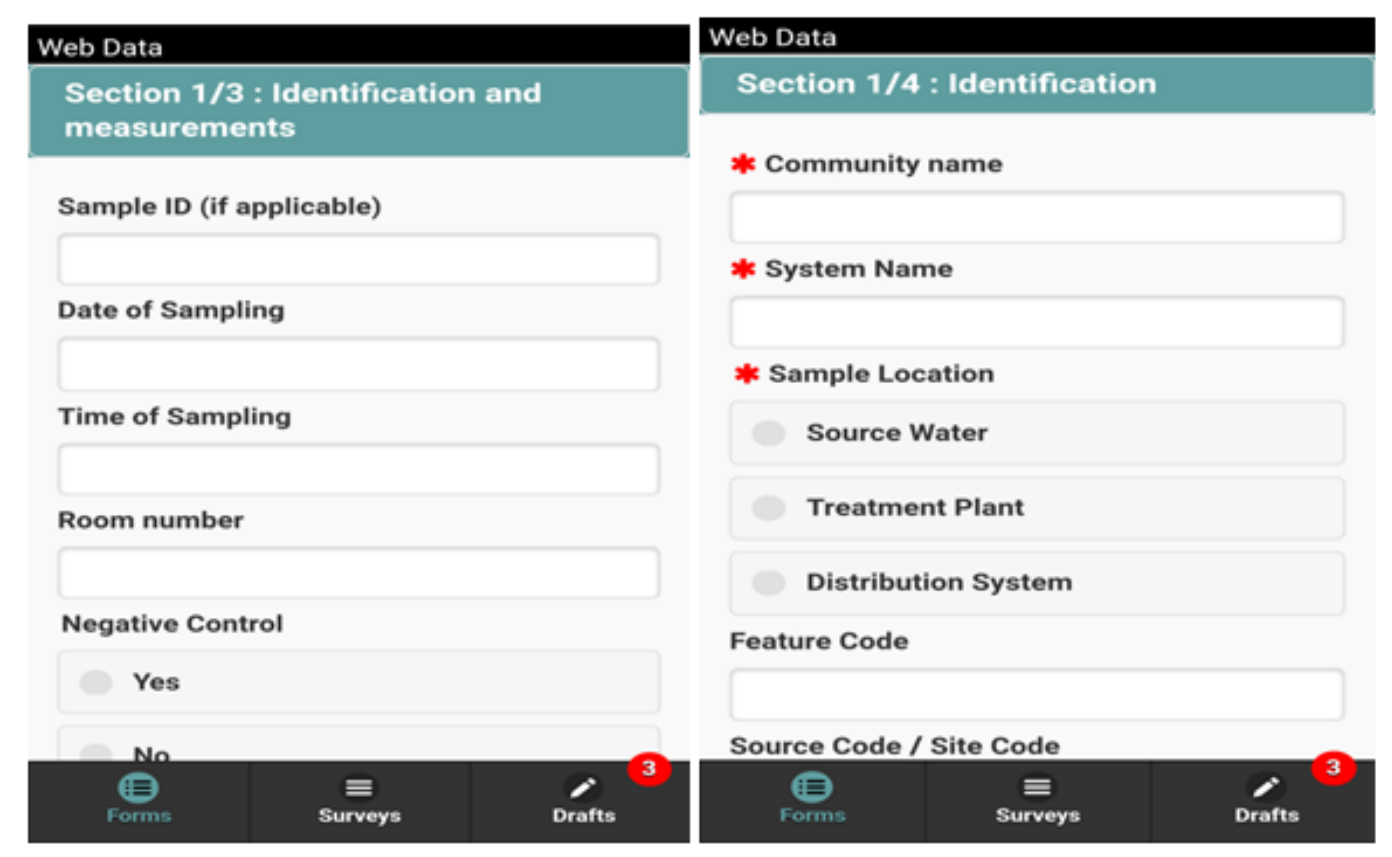

a) Form for SRI pilot b) Form for FNIHB-MB pilot

Figure 3: Data collection form that was developed for a) SRI and b) FNIHB-MB pilot

The next step was to identify participants for both pilots who were provided appropriate access to the CNPHI platform in order to gain access to the forms. The participants were provided with a user guide to download and use the app. Instructions were included on accessing the form on the app and downloading it for offline use. Information was also provided on special features, such as setting-up a pin for offline data collection, and the limitations of the app during the pilot phase.

A total of 146 records were collected using the mobile platform from both pilots. During the course of the pilots, feedback was collected as per CNPHI's agile philosophy for application development. Thus, any issues or bugs were addressed and rectified on an ongoing basis. Moreover, participants were also informed when a new version of the app was released or when any modifications were made to any app features. The submitted records were instantly available through the web platform for interactive interrogation and further analysis. Instant capabilities included, data queries and analysis, GIS mapping and cluster analysis.

\section{Evaluation of the CNPHI on the go app}

As per CNPHI's agile approach to application development, user feedback and comments were submitted by participants to CNPHI during the entire pilot phase on a routine basis. Some of the 
issues encountered during the pilot included: issues with log-in (a longer than usual time to login); incorrect GPS coordinates in the records; issues with offline data collection; and incorrect dates in some records. Using CNPHI's philosophy to development provided an ability to implement fixes and changes on an ongoing basis in partnership with users.

A questionnaire was designed and sent to pilot leads at the conclusion of each pilot to explore the strengths and limitations of the application, to capture user experience and to compare and contrast their prior data management approaches with CNPHI on the go.

\section{Results}

\section{Pilot 1: FNIHB - MB}

The FNIHB-MB Drinking Water Safety Program uses sensors and test units to monitor water quality and collect relevant information across First Nations Communities in Manitoba. As part of this program, field personnel, such as community based water monitors, collect and analyze water sample results locally in a laboratory that is set up in the community. After this step, results are reported to the EHO. In addition, EHOs will also take water samples for submission to accredited labs and collect additional information from the field during site visits or follow-up inspections. Current practice involves recording of sample information and associated results using a paper form, which is faxed to the central office for review by EHO's and then manually entered into a database. This places a significant administrative burden on personnel and introduces delays in the process. It was mentioned that there can be significant lags between collection and receipt of water sampling and other monitoring data from the utilities. Importantly, it was recognized that the flow of key information that can provide early warning of changing conditions, a decline in water quality or a loss of control in a drinking water system is subject to a significant lag. The pilot helped to highlight the need for improvements to the flexibility and timeliness of field data collection and the need for more agile generation of intelligence in support of risk management decisions and public health protection.

Through stakeholder discussions, other drinking water agencies also identified similar challenges related to a lack of timely collection and interrogation of field data. Due to this, there is a clear need to modernize the overall data collection and reporting process in regards to drinking water safety programs. Having an app to record and submit sample results would accelerate data submission and the availability of results, alleviating the workload and time required for manual entry. In addition, concurrent readings from the field related to key operational controls, such as chlorine residuals or turbidity fluctuations, better equip decision-makers to implement more timely health protection measures whenever the safety of drinking water is compromised or threatened.

\section{Pilot 2: Sunnybrook Research Institute}

The traditional process for data collection employed by SRI involves manually writing sample information on a paper form, then transferring the information into an Excel document. As additional data becomes available (including laboratory results), it is also entered manually into the excel document. Information sharing is achieved through the use of encrypted excel documents sent via e-mail. 
Benefits of using CNPHI on the go in the field included efficiency, enhanced biosafety through the reduction in fomites though the elimination of notepaper and writing implements, and realtime access to data with analytics. Minor issues were resolved within very short timeframes, thus enabling ongoing data collection and analysis without delay.

Both SRI and FNIHB-MB pilots reported an overall positive experience of using the app. Some of the key findings include:

- CNPHI on the go allowed seamless data collection while at the sampling site, followed by seamless data management on the web platform, and rapid information sharing for anyone with appropriate access. It made the entire process much simpler, less time-consuming, and overall fewer steps were required for data collection and management. It also allowed for quick data extraction when required.

- A major benefit is the ability to collect data without an active internet connection. Further, significant efficiencies were gained by directly entering information on the app without having to write it down or subsequently transfer it to an electronic system. Integration of the web and mobile platforms for secure information sharing between multiple involved parties provides a key benefit for rapid data collection and sharing. Both programs see the application being used on a regular basis for the collection of monitoring and surveillance data where smartphones are used.

\section{Discussion}

Based on the results of the two pilots, the CNPHI on the go app provides a significant opportunity for rapid data collection from the field. Further, through its integration with the existing CNPHI web-based platform, the technologies are positioned to enhance existing initiatives across various health protection disciplines, already well evolved on CNPHI.

Specifically, the technology can provide the following key benefits:

- Remote data collection: The app allows users to collect public health data in remote locations without Wi-Fi or cell reception.

- Turnaround time: The turnaround time of the data collection is near-real time, allowing decision-makers to access the data instantly and use tools that allow for querying, analysis, visualization and intelligence generation.

- Precision: There is a reduction in transcription errors given that the data does not need to be manually entered from paper into a database.

- Secure data: Access to the app requires CNPHI credentials and follows the same security standards including built-in user audit functions.

- Efficiency: The data collection process requires less human resource commitment resulting in administrative cost savings.

- Easy and simple to use: The app is designed to be easy and simple to use. Moreover, additional features such as the 4 digit pin allow users to quickly log-in without needing to enter the username and password. 
- Flexibility: The Web Data technology provides an ability to interactively develop forms, providing flexibility to non-technical users for rapidly developing secure forms for data collection. It also allows administrators to modify the forms once deployed or create new forms as needed.

- Multiple types of data: Data fields include; text, pick lists, checkboxes, radio buttons and numbers, allowing various types of information to be collected using the mobile web data technology.

- Early Warning: Faster access to data improves not only analysis and program responsiveness, but improves the ability to react to unexpected changes. Moreover, since the data collection forms can be customized, further questions can be added to investigate interesting or critical findings in the field.

- Customizable: In contrast to a purpose-built approach, the CNPHI on the go platform is customizable (flexible by design) allowing for dynamic and rapid deployment based on the need.

- Analysis: Web Data provides administrators with integrated search and interrogation capabilities. It also provides the ability for multiple users to access records at the same time while allowing for the production of instant data reports.

It is important to note that although the platform enables a completely dynamic capability for data collection, avoidance of collection of personal or sensitive information is respected across the platform.

\section{Conclusion}

The CNPHI on the go is well poised to uniquely address the challenges related to rapid mobile data collection by enabling rapid form development and distribution, online and offline data collection, secure access to the data, and interactive data analysis. Some of the tools on the CNPHI platform, such as, the Response Centre technology have also been extended to the mobile app. This provides a capability for a two-way exchange of information between field personnel and administrators, allowing tasks and information to be managed in an efficient manner. Moreover, a potential addition of other tools within the CNPHI web platform would provide an infrastructure to support rapid data collection, bi-directional communication, investigations, outbreak management and the generation of geo-aware bio-surveillance intelligence related to public health issues of concern including human, animal, and environmental health. It will provide tools allowing close to real-time addition of intelligence related to the entire spectrum of surveillance, prevention, preparedness and response to events potentially affecting the health of the Canadian public.

Some of the potential applications of the mobile technology include:

- Support for outbreak investigations: Data for outbreak investigations could be collected in the field using mobile technology and submissions can be done in near-real time, which would provide the outbreak response team with quick access to data prompting quick analysis and response.

- An inventory tool for key resources: The technology could also be used as an inventory management tool for validating and managing the working conditions of emergency kits. 
- Support for active or passive surveillance (e.g., collecting data on cases with influenza-like illness at monitoring clinics; a screening tool to monitor travel related illness by quarantine officers at ports of entry).

- Emergency management: During emergencies (e.g., wild fires, floods or extreme weather events) field activities and information can be managed under rapidly changing conditions. Field personnel can be tasked and can respond with information while their safety and status can be monitored.

\section{Limitations}

One of the limitations is that the current version of the app is only designed for Android operating systems. However, there are plans to expand to other operating systems in the future.

Additionally, sample size for pilot participation was small for capturing the overall readiness of public health professionals to use such a platform on a regular basis and with full integration into surveillance activities. Further pilots and testing have been planned to support informed ongoing evolution of the technology.

\section{Acknowledgements}

We would like to acknowledge that the pilots were supported by funding from the Canadian Safety and Security Program (CSSP), managed through Defence Research and Development Canada (DRDC) Centre for Security Science (CSS). Authors would like to thank the contributions of the CNPHI team, Karren Prost and EHO's from FNIHB - MB for their support and input throughout the pilots.

\section{References}

1. Radio-Television, C., \& Telecommunications Commission. (2017). Communications Monitoring Report 2017. Canadian Radio-television and Telecommunications Commission.

2. World Health Organization. (2011). mHealth: new horizons for health through mobile technologies. mHealth: new horizons for health through mobile technologies.

3. Mahundi M, Kaasbøll J, Twaakyondo H. (2011). Health information systems integration in Tanzania: tapping the contextual advantages. In IST-Africa Conference Proceedings, 2011 (pp. 1-11). IEEE.

4. Mukhi S, Aramini J, Kabani A. 2007. Contributing to communicable diseases intelligence management in Canada: CACMID meeting, March 2007, Halifax, Nova Scotia. Can J Infect Dis Med Microbiol. 18(6), 353-56. PubMed https://doi.org/10.1155/2007/386481 
5. Mukhi SN, Chester TLS, Klaver-Kibria JD, Nowicki DL, Whitlock ML, et al. 2011. Innovative technology for web-based data management during an outbreak. Online J Public Health Inform. 3(1). PubMed https://doi.org/10.5210/ojphi.v3i1.3514

6. Kloeze H, Mukhi SN, Alexandersen S. 2013. Swine influenza test results from animal health laboratories in Canada. Can Vet J. 54(5), 501. PubMed 